\title{
Особенности метрологического обеспечения оптических рефлектометров
}

В. В. Григорьев, В.Е. Кравцов, А. К. Митюрев , Е.А. Науменко, А. О. Погонышев, К. Б. Савкин, С. В. Тихомиров ФГУП «ВНИИОФИ», www. vniiofi.ru,

${ }^{1}$ mak@vniiofi.ru, Москва, Россия

В статье представлены результаты исследовательских работ, направленных на обеспечение единства измерений параметров оптических рефлектометров. Описываются эталонная база и нормативно-техническая документация, созданные специалистами ВНИИОФИ. Рассматриваются результаты внедрения и применения созданных эталонов. Также приводятся результаты исследований методов калибровки нового перспективного класса оптических рефлектометров, работающих в частотной области.

Ключевые слова: метрологическое обеспечение, оптический рефлектометр, импульсное лазерное излучение

\section{Features of Optical Reflectometers Metrological Support}

V.V.Grigoriev, V.E. Kravtsov, A. K. Mityurev', E. A. Naumenko, A. O. Pogonyshev, K. B. Savkin, S. V. Tikhomirov The All-Russian Research Institute for Optical and Physical Measurements, www. vniiofi.ru,

${ }^{1}$ mak@vniiofi.ru,Moscow, Russia

\begin{abstract}
The results of optical time domain reflectometer (OTDR) metrological support activities are presented. Metrological and normative standards in the field are described. OTDR metrological standards implementation and exploitation are considered. The results of calibration methods research for optical frequency domain reflectometer (OFDR) are also presented.
\end{abstract}

Keywords: metrological support, OTDR, OFDR, pulsed laser radiation

дним из приборов, обеспечивающих диагностику волоконно-оптических систем передачи информации и других волоконно-

* Примечание редактора: Статья рекомендована программным комитетом конференции, проходившей в рамках специализированной выставки лазерной, оптической и оптоэлектронной техники «Фотоника. Мир лазеров и оптики. 2019». оптических систем, являются оптические рефлектометры (ОР). Они позволяют измерять длину оптических волокон и кабелей (ОВ, ОК), расстояние до места неоднородности и ослабление в ОВ и OK при производстве и эксплуатации систем и их компонентов. Во ВНИИОФИ, государственном научном метрологическом институте в области оптико-физических измерений, проводятся 
работы, направленные на обеспечение достоверности результатов и единства измерений указанных параметров, что необходимо для достижения высокого качества связи и передачи информации. С этой целью для решения задач метрологического обеспечения измерений параметров ОР, работающих во временной области (OTDR), разработаны Первичный и Рабочие эталоны, а также соответствующая нормативная документация.

В 2006 году был создан, а затем в 2011 году усовершенствован Государственный первичный специальный эталон ГЭТ 170-2011 (ГПСЭ) и разработана межгосударственная поверочная схема ГОСТ 8.585-2013 [1], регламентирующая передачу единиц от верхнего звена - первичного эталона, к рабочим эталонам и средствам измерений. ГПСЭ возглавляет данную поверочную схему. Воспроизведение и передача единицы длины в ГПСЭ производится путем определения значений времени задержки оптического излучения с последующим пересчетом к значениям длины. Воспроизведение и передача единицы ослабления производится путем нормирования относительных значений мощности излучения. В настоящее время с целью подтверждения характеристик первичного эталона проходят международные сличения по длине оптического волокна в рамках проекта APMP.PR-S8, участниками которого являются такие страны, как Республика Корея, Франция, Швейцария, Китай и др.

Созданные Рабочие эталоны, обеспечивающие поверку и калибровку OP (OTDR), основаны на использовании активного метода [2, 3] передачи единиц длины и ослабления. С этой целью в составе Рабочих эталонов применяются специальные оптические генераторы, имитирующие прохождение оптического излучения по волокну, разработанные совместно с Институтом информационных технологий (Минск, Республика Беларусь). Относительная погрешность воспроизведения единицы ослабления с помощью разработанных Рабочих эталонов для одномодового режима не превышает 0,015 дБ/дБ, а абсолютная погрешность воспроизведения единицы длины не превышает $\left(0,15+5 \cdot 10^{-6} \cdot \mathrm{L}\right), \mathrm{M}$, где $\mathrm{L}-$ значение длины, м.

K настоящему моменту ВНИИОФИ поставил более 40 Рабочих эталонов для оснащения центров стандартизации и метрологии и ряда ведомственных метрологических центров.

С помощью созданных эталонов во ВНИИОФИ проведены испытания и включены в реестр более 60 типов ОР, произведенных зарубежными фирмами (EXFO, Agilent, Viavi, Yokogawa и рядом других) и отечественными компаниями (ТПК ВП, Связьприбор, Связьсервис). ВНИИОФИ также выполняет поверку и калибровку рефлектометров (более 200 приборов за 2017 год). Для обеспечения поверки разработаны Рекомендации по методам поверки Р 50.2.071-2009 [4].

Следует отметить, что подавляющая часть поверяемых приборов сохраняет свои характеристики и успешно проходят поверку. Вместе с тем отмечаются случаи, когда погрешности при измерении длины и ослабления превышают допустимые пределы, происходят сбои в программном обеспечении и уменьшается динамический диапазон вследствие неправильного обращения с разъемами и деградации лазеров; в ряде случаев отмечяется сдвиг по шкале длины, что требует пристального внимания со стороны эксплуатирующих организаций и подтверждает необходимость работ по периодическому контролю основных технических характеристик OP.

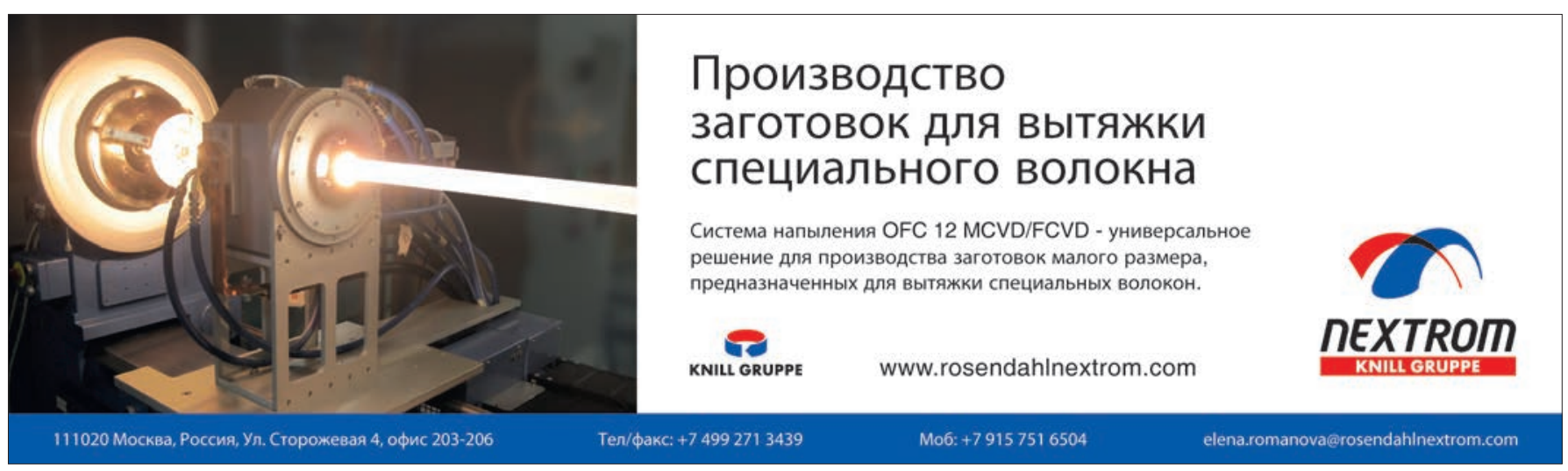


В настоящее время с развитием высокоскорост ных систем и оптических систем доступа (волокно в каждый дом) возникает необходимость применения ОР с набором источников, работающих на большем, чем традиционно, количестве длин волн и с погрешностями, приближающимися к погрешностям Рабочих эталонов. Возникает также необходимость нормирования значений обратных потерь [3]. Все это требует постоянного совершенствования эталонной базы.

Одним из таких перспективных классов ОР являются OFDR рефлектометры (Optical Frequency Domain Reflectometry), отличительной особенностью которых является сочетание быстродействия и высокого разрешения порядка 10 мкм при измерении коротких расстояний до нескольких десятков метров.

Принцип работы OFDR рефлектометров основан на методике частотной интерферометрии [4], позволяющей получать рефлектограммы с высоким пространственным разрешением и значительным динамическим диапазоном. Преимуществом OFDR является также отсутствие мертвой зоны при измерении расстояний.

В настоящее время в России отсутствует метрологическая база, позволяющая обеспечить поверку, калибровку и испытания данного типа приборов. Кроме того, имеется ряд методических вопросов, связанных с отсутствием алгоритмов и количественных оценок по определению погрешности приборов, для которых, как правило, в спецификации указывается лишь параметр пространственного разрешения и не указываются соответствующие точностные характеристики .

Был проведен анализ принципиальной схемы типичного прибора $[4,5]$, в результате которого построена математическая модель процесса преобразования сигналов в OFDR рефлектометре. B модели имитируется подключение к прибору тестируемого ОВ с неоднородностями, положение которых требуется определить. Интерференционный сигнал, зависящий от местоположения отражающих событий, описывается с помощью известных аналитических соотношений $[4,5]$, находится обратное преобразование Фурье полученного сигнала, и в результате получаются искомые временные задержки, то есть определяется положение неоднородностей в тестируемом ОВ. Полученные с помощью математической модели значения абсолютной погрешности определе- ния положения неоднородностей в тестируемом волоконно-оптическом тракте для типовых приборов составляют порядка 15 мкм при длине линии до 30 м и 30 мкм при длине линии до 70 м.

В зависимости от характеристик рассматриваемых приборов для обеспечения калибровки OFDR рефлектометров с требуемой погрешностью предложены следующие методы: калибровка газовой кюветы прибора по длине волны, интерферометрический метод, калибровка с помощью компаратора линейных перемещений, калибровка с помощью Государственного первичного специального эталона ГЭТ 170-2011 [1].

Метод калибровки газовой кюветы прибора по длине волны заключается в определении длин волн пиков поглощения кюветы и оценке отличия полученных значений от опорных (эталонных), используемых прибором для вычислений длины. Данный метод применим лишь в том случае, когда имеется доступ к газовой кювете прибора и настройкам его программного обеспечения в части установки значений длин волн пиков поглощения .

Калибровка интерферометрическим методом производится с помощью образцов оптического волокна, длина которых с высокой степенью точности определяется с использованием интерферометра и перестраиваемого по длине волны лазера, а затем измеряется на калибруемом OFDR рефлектометре.

Идея метода калибровки OFDR рефлектометров с помощью компаратора линейных перемещений заключается в применении высокоточной оптической линии задержки с параметрами, определяемыми по результатам измерений перемещений интерференционным методом.

Калибровка с помощью государственного первичного специального эталона ГЭТ 170-2011 производится с помощью образцов ОВ, время задержки прохождения оптического сигнала, в котором с высокой степенью точности определяется с помощью генератора временных интервалов из состава ГэТ 170-2011, с последующим измерением их длин на калибруемом OFDR рефлектометре. Данный метод целесообразно использовать для относительно больших расстояний - порядка двух километров.

Кроме задач, связанных с метрологическим обеспечением рефлектометрии OTDR и OFDR, для диагностики волоконно-оптических систем и устройств все более широкое применение нахо- 
дят другие классы ОР и измерительных систем с распределенными параметрами. К ним относятся когерентные рефлектометры, системы на основе Брюллюэновского и Рамановского рассеяния и др. Для них также существенным является нормирование расстояния до места события. При этом в ряде систем требования по точности измерений расстояний определяются значениями порядка десятков микрон. В связи с этим во ВНИИОФИ проводятся работы по испытаниям и калибровке нескольких типов распределенных волоконно-оптических систем. Начаты также работы по методам калибровки и поверки рефлектометров OFDR и когерентных OP

Таким образом, разработанная в настоящее время система метрологического обеспечения измерений с помощью OP OTDR, включающая Государственный первичный специальный эталон, Рабочие эталоны и нормативную документацию, обеспечивает выполнение необходимых работ по метрологическому обеспечению измерений их основных параметров.

Дальнейшие работы в данной области предполагается проводить в направлении увеличения точности эталонов, расширения спектральных диапазонов и создания эталонной базы для вновь применяемых перспективных типов ОР.

\section{СПИСОК ЛИТЕРАТУРЫ}

1. ГОСТ 8.585-2013. Государственная поверочная схема для средств измерений диины и времени распространения сигнала в световоде, средней мощности, оспабления и дпины вопны оптического изпучения дпя волоконно-оптических систем связи и передачи информации. - Введ. 01.01.2015. - М.: Стандарт информ, 2015.

GOST 8.585-2013. Cosudarstvennaya poverochnaya skhema dlya sredstv izmerenij dliny i vremeni rasprostraneniya signala v svetovode, srednej moshchnosti, oslableniya i dliny volny opticheskogo izlucheniya dlya volokonno-opticheskih sistem svyazi i peredachi informacii. - Vved. 01.01.2015. - M.: Standart inform, 2015

2. Григорьев В. В., Кравцов В. Е., Митюрев А. К., Мороз Е. А., Погонышев А. О., Подюкова Л. В., Савкин К. Б., Тихомиров С. В. Современные оптические рефлектометры: вопросы метрологического обеспечения (обзор). Фотон-экспресс. 2018; 5: 18.

Grigor'ev V.V., Kravcov V. E., Mityurev A. K., Moroz E. A., Pogonyshev A. O., Podyukova L. V., Savkin K. B., Tihomirov S. V. Sovremennye opticheskie reflektometry: voprosy metrologicheskogo obespecheniya (obzor). Foton-ekspress. 2018; 5: 18

3. IEC61746-1 Ed. 1 OTDR Calibration OTDR for SM fibers.

4. Soller B. J., Wolfe M., Froggatt M. E. Polarization resolved measurement of Rayleigh backscatter in fiber-optic components. National Fiber Optic Engineer's Conference, OSA Technical Digest Series (Optical Society of America, Washington, DC), paper NWD3 (2005);

5. Kreger S. T., Gifford D. K., Froggatt M. E., Sang A. K., Duncan R. C., Wolfe M. S., Soller B. J. High-Resolution Extended Distance Distributed FiberOptic Sensing Using Rayleigh Backscatter. SPIE. 2007; 6530: 65301R.

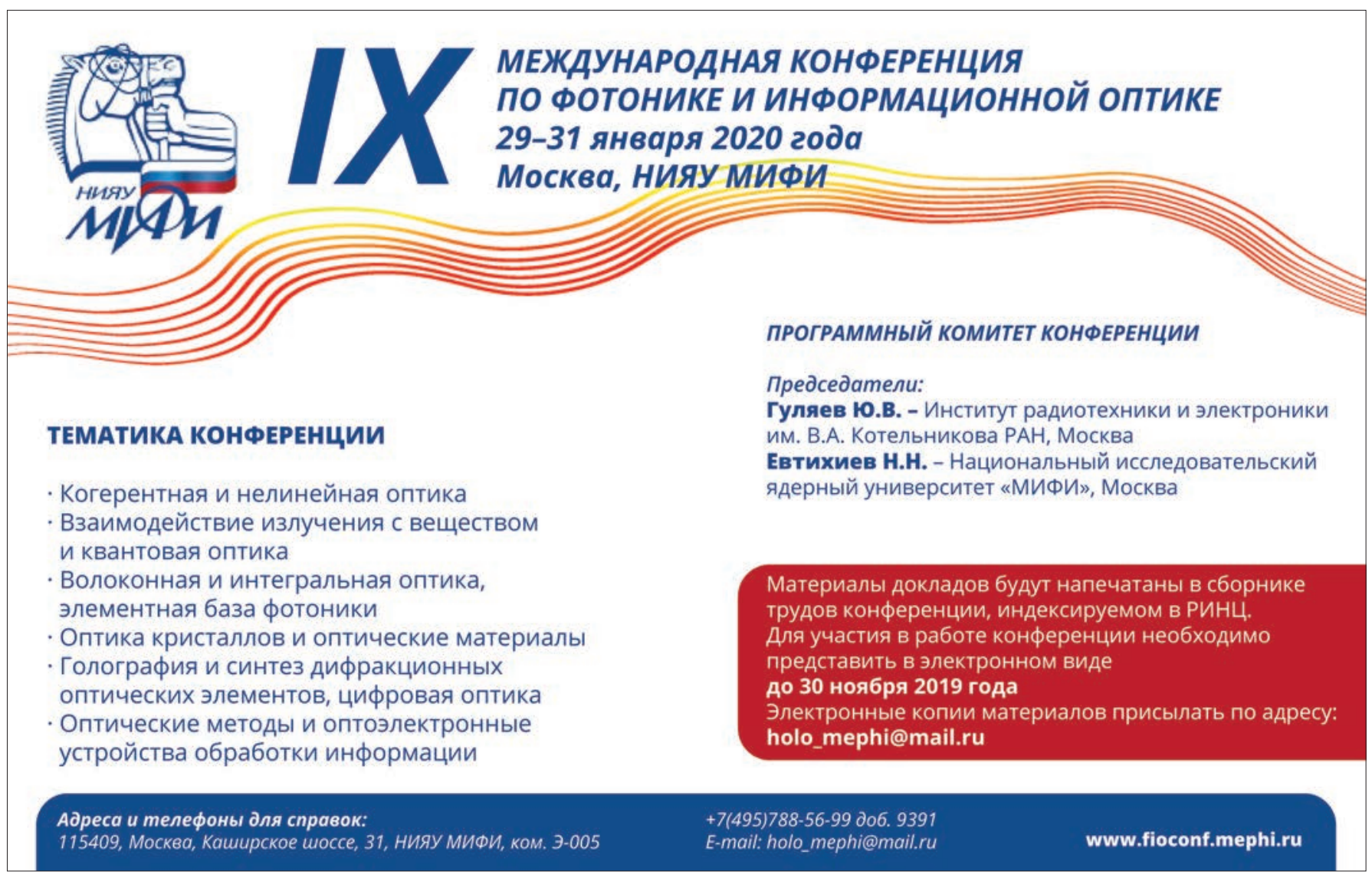

